Ann. Génét. Sél. anim., I979, 11 (4), 397-4I2.

\title{
Gène cou nu et performances de croissance selon la température chez le poulet
}

\author{
L. E. MONNET, A. BORDAS, P. MÉRAT \\ Laboratoire de Génétique Factorielle, \\ Centre National de Recherches Zootechniques, I.N.R.A., \\ 78350 Jouy-en-Josas, France
}

\begin{abstract}
Résumé
La croissance de poulettes des génotypes $\mathrm{Na} N a$ (homozygote "cou nu "), $\mathrm{Na}$ na (" cou nu » hétérozygote) et na na (plumage "normal ") a été comparée en poussinière au sol, a une température ambiante d'environ $20^{\circ} \mathrm{C}$ après l'âge de 4 semaines (lot témoin), ou à $3{ }^{\circ}{ }^{\circ} \mathrm{C}$ jusqu'à Io semaines (lot chauffé). Une comparaison similaire à $31^{\circ} \mathrm{C}$ a été faite sur des coquelets des mêmes génotypes en cages individuelles. Le poids corporel individuel était mesuré toutes les deux semaines, ainsi que la consommation alimentaire, globalement par génotype pour les femelles ou individuellement pour les mâles. Chez ces derniers, la longueur des barbillons et des tarses, la température rectale et le poids des plumes étaient enregistrées à Io semaines. Le plumage était pesé à des âges successifs chez un autre groupe de coquelets.

Le poids du plumage, absolu ou ramené au poids corporel, diffère significativement pour les trois génotypes. Son accroissement relatif avec l'âge paraît moins marqué pour les poulets " cou nu ". La croissance corporelle jusqu'à ro semaines ne diffère pas significativement chez les poulettes dans le lot témoin, mais la consommation alimentaire est plus élevée et l'indice deconsommation moins bon pour le génotype $\mathrm{Na} N a$. En lot chauffé, l'homozygote $\mathrm{Na} N a$ a sur le génotype na na un avantage de croissance à ro semaines respectivement d'environ I I et Io p. Ioo chez les femelles et les mâles, et, de même, l'hétérozygote a, dans les deux sexes, un avantage voisin de $9 \mathrm{p}$. roo. La consommation d'aliment est plus élevée pour les animaux " cou nu ", et l'efficacité alimentaire est voisine pour les trois génotypes dans ces conditions. La température rectale à Io semaines en lot "chauffé " est légèrement inférieure chez les poulets $\mathrm{Na} \mathrm{Na}$ ou $\mathrm{Na}$ na que chez les na na. L'ensemble de ces résultats indique que la réduction de l'emplumement par le gène $\mathrm{Na}$ améliore la thermotolérance en période de croissance. Ceci, ajouté au rendement à l'abattage meilleur pour les poulets $\mathrm{Na} N a$ ou $\mathrm{Na} n a$ comparés aux na na (respectivement d'environ 3 et I,5 p. I oo) du fait de la réduction du plumage, suggère que dans des conditions de température analogues à celles de notre lot chauffé, l'introduction du gène " cou nu " dans des populations destinées à la production du poulet de chair pourrait être avantageuse.
\end{abstract}

\section{Introduction}

Le gène $\mathrm{Na}(\mathrm{Cou} n u)$ réduit chez la poule domestique le plumage et l'extension des ptérylies (HUT'T, I949), avec dominance incomplète (CRAWFORD, r976; ScoTT et CRAWFORD, I977). A température modérée, il a une très légère in fluence dépressive sur le poids corporel des mâles à 8 semaines d'âge, probablement explicable 
par la réduction du plumage (MERAT, I979). En réponse à un stress thermique, SMITH et LEE (I977) obtiennent moins de mortalité pour des poussins hétérozygotes $N a$ na que pour ceux de génotype na na. En l'absence de données publiées sur les performances de croissance associées à ce gène à température élevée, nous avons comparé des coquelets des trois génotypes $N a \mathrm{Na}, \mathrm{Na} n a$ et na na, élevés à $3 \mathrm{I}^{\circ} \mathrm{O}$ en cages individuelles de o à Io semaines (Bordas et al., I978). La présence du gène $N a$ s'accompagnait d'un avantage très appréciable de croissance dans ces conditions, avec un indice de consommation peut-être amélioré. Le présent article confirme ces résultats et les étend aux poulettes.

\section{Matériel et méthodes}

\section{I. - Matériel animal et période pré-expérimentale}

Les poussins expérimentaux sont issus de parents tous deux hétérozygotes $N a$ na (9 pères au total et 4 à 8 mères par père). Ces parents, élèves à Jouy-enJosas, étaient les descendants $\mathrm{F}_{1}$ d'un croisement ơ na na $\times$ o $\mathrm{NaNa}$ fait à l'Élevage de Las Costes, comme dans la précédente expérience (BoRDAs et al., r978).

Les poussins provenaient de deux éclosions pedigree espacées de $2 \mathrm{I}$ jours, la première ayant eu lieu le 26 septembre 1978 . A 1'éclosion, le sexe et le génotype ( $N a N a, N a$ na ou na na) étaient déterminés. La première éclosion (mâles et femelles) devait fournir, pendant la période expérimentale ultérieure (4 à ro semaines d'âge). le lot "témoin ", la seconde éclosion donnant le lot "chauffé " (cf. $\S 2$ ).

A I jour d'âge, les poussins destinés au lot "témoin ", étaient placés dans une poussinière au sol, avec une température initiale sous éleveuse voisine de $35^{\circ} \mathrm{C}$, diminution graduelle, puis, après 4 semaines, maintien de celle-ci autour de $20^{\circ} \mathrm{C}$, conformément aux normes habituelles.

Pour le lot chauffé, les femelles étaient mises dans une poussinière identique, et la température du local, à partir de la deuxième semaine d'âge, était maintenue aux environs de $3 I^{\circ} \mathrm{C}$. Quant aux poussins mâles, ils étaient distribués par groupes de 4 dans 60 cages dans un local conditionné en température, avec surpression par pulsion d'air chaud. Un seul individu était gardé par cage à partir de 2 semaines d'âge (voir § 2). La température initiale était de $34^{\circ} \mathrm{C} \pm \mathrm{I}^{0}$, avec un degré hygrométrique voisin de $3^{0} \mathrm{p}$. Ioo, puis était abaissée jusqu'à $3^{\mathrm{I}^{\circ} \mathrm{C}} \pm \mathrm{I}^{0}$ à partir de la $2^{\mathrm{e}}$ semaine de façon analogue aux femelles.

Tous les lots recevaient de o à Io semaines I4 heures de lumière et Io heures d'obscurité par jour. L'eau et l'aliment étaient donnés ad libitum, ce dernier sous forme de farine à I $8 \mathrm{p}$. Ioo de protéines totales et $2800 \mathrm{Kcal} / \mathrm{kg}$ d'énergie métabolisable ( $\mathrm{rr}, 78 \mathrm{MJ} / \mathrm{kg}$ ).

\section{2. - Conditions expérimentales}

La période expérimentale dure de 4 à ro semaines d'âge. La détermination des conditions dans chaque lot tient compte des contraintes matérielles et du fait que les performances de croissance en lot " non chauffé " étaient déjà connues (MÉRAT, I979), pour les génotypes $N a$ na et na na. 
a) Femelles, lot " témoin"

A 4 semaines, pour chaque génotype $(N a N a, N a n a, n a n a)$, un échantillon de même effectif ( $n=55$ à 2 semaines) est gardé sur la base du poids à 2 semaines d’âge de façon à homogénéiser le poids moyen des trois groupes et à obtenir un dispositif factoriel génotype $\times$ famille de père le plus équilibré possible. Les trois génotypes sont relogés au sol dans 3 cellules distinctes, séparées par une cloison grillagée. L'éclairage est assuré par 2 ampoules de 75 watts supplémentant la lumière du jour. Les températures maximales et minimales par semaine sont indiquées au tableau I. L'hygrométrie n'est pas contrôlée; elle fluctue autour de $25 \mathrm{p}$. Ioo durant la période considérée.

En fin de période expérimentale, les effectifs sont respectivement $52,5^{2}$ et 54 pour les génotypes $N a N a, N a$ na et na na.

\section{TABLEAU I}

Températures d'élevage du lot "témoin " et du lot "chauffe " Ambient temperatures for the "control " and " heated " groups

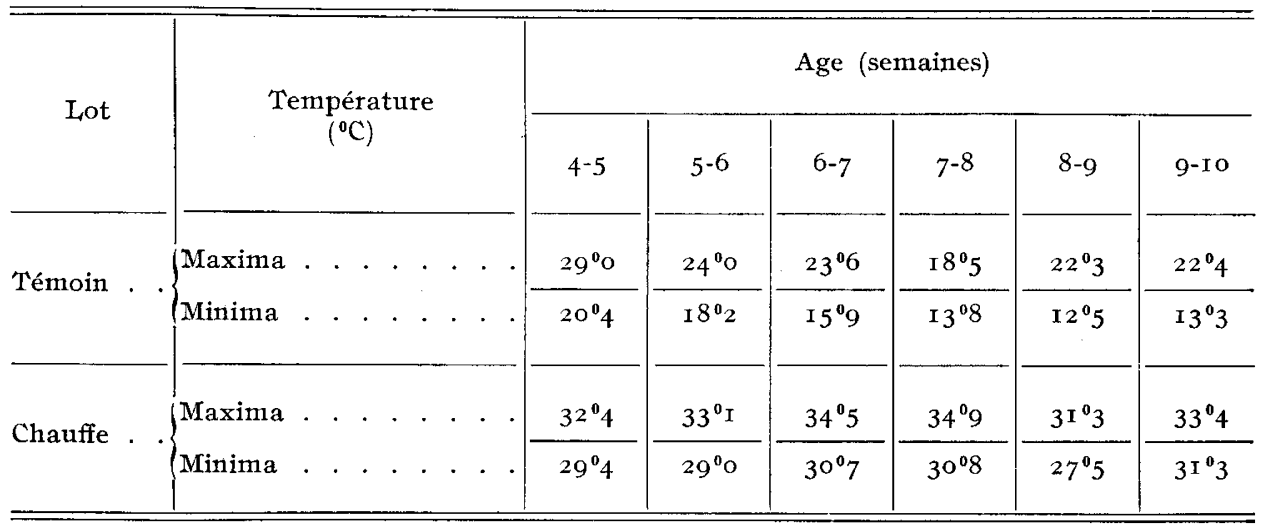

b) Mâles, lot " témoin"

Les conditions d'élevage sont analogues à celles des femelles, mais les 3 génotypes sont élevés ensemble dans une cellule unique. Ce lot est utilisé uniquement pour des pesées du plumage âge par âge (§ 3) et n'intervient pas dans les comparaisons relatives à la croissance.

\section{c) Femelles, lot "chauffé "}

Comme pour les femelles du lot " témoin ", une pesée à 2 semaines d'âge permet de constituer trois échantillons (chacun de 55 individus) homogènes pour le poids moyen, correspondant aux 3 génotypes et élevés au sol séparément. Seuls la température ambiante et le mode d'éclairement diffèrent : température moyenne variant autour de $3 \mathrm{I}^{\circ} \mathrm{C}$ (tab1. I), cellules sans fenêtres éclairées par 2 ampoules de $75 \mathrm{~W}$. En fin d'expérience, les effectifs sont respectivement 55,55 et 53 pour les génotypes $N a N a, N a n a$ et na na. 
d) Mâles, lot "chauffé "

Après la pesée à 2 semaines, 20 coquelets par génotype, issus de 5 familles de pères, formant trois groupes homogènes pour le poids moyen, sont choisis et distribués au hasard dans les 60 cages mentionnées plus haut. La température est maintenue à $3^{\mathrm{I}}{ }^{\circ} \mathrm{C} \pm \mathrm{I}^{0}$. L'éclairage est assuré par des tubes fluorescents. Le degré hygrométrique n'est pas contrôlé et varie comme pour les femelles.

\section{3. - Critères mesurés}

Les observations et les symboles et unités employés sont résumés au tableau 2.

TABLEAU 2

Observations et unités employées

Recorded traits and units

\begin{tabular}{|c|c|c|}
\hline $\begin{array}{l}\text { Abreviation } \\
\text { (Abbreviation) }\end{array}$ & $\begin{array}{l}\text { Rubrique } \\
\text { (Item) }\end{array}$ & $\begin{array}{l}\text { Unité } \\
(\text { Unit })\end{array}$ \\
\hline$n$ & Effectif (Number) & \\
\hline $\mathrm{P}$ & $\begin{array}{l}\text { Poids vif individuel à l'âge indiqué (Individual body weight at } \\
\text { specified age) }\end{array}$ & $\mathrm{g}$ \\
\hline$\Delta \mathrm{P}$ & $\begin{array}{l}\text { Variation de } \mathrm{P} \text { au cours de la période indiquée (Body weight varia- } \\
\text { tion in the specified period) }\end{array}$ & $g$ \\
\hline $\mathrm{O}$ & $\begin{array}{l}\text { Quantité d'aliment consommée pour la période (Food consumed } \\
\text { during the period) }\end{array}$ & g \\
\hline I & $\begin{array}{l}\text { Indice de conversion alimentaire }=\mathrm{O} / \Delta \mathrm{P} \text { (Food conversion }= \\
\quad \mathrm{O} / \Delta P \text { ) } \\
\text { Longueur des barbillons (Wattle length) } \\
\text { Longueur du tarse (Shank length) } \\
\text { Température rectale (Rectal temperature) } \\
\text { Poids du plumage (Weight of plumage) } \\
\text { Poids du plumage en p. cent du poids corporel (Weight of plumage } \\
\text { in p. roo of body weight) }\end{array}$ & $\begin{array}{l}\mathrm{mm} \\
\mathrm{mm} \\
{ }^{\circ} \mathrm{C} \\
\mathrm{g}\end{array}$ \\
\hline
\end{tabular}

Poids vif

Les poussins (sauf les mâles du lot " témoin "), sont pesés individuellement à $2,4,6,8$ et ro semaines d'âge. On en déduit la variation de poids individuelle par périodes de 2 semaines. 


\section{Consommation et efficacité alimentaire}

Pour les poulettes des lots " témoin " et "chauffé ", élevées au sol, on ne dispose que de la quantité globale d'aliment consommé par génotype, par périodes de 2 semaines jusqu'à ro semaines d'âge. Il en est de même de l'efficacité alimentaire (rapport du poids d'aliment consommé au gain de poids pour une période donnée). Pour les coquelets du lot "chauffé ", la consommation est enregistrée individuellement, ce qui permet d'établir des indices de conversion individuels.

\section{Développement pondéral du plumage}

Le poids total des plumes est mesuré à 4, 6, 8 et ro semaines d'âge sur 7 coquelets du lot " témoin " par génotype et par âge, les coquelets des différents génotypes à chaque âge étant frères ou demi-frères par groupes de trois. Ils sont mis à jeun la veille, abattus et saignés, pesés, plumés, puis pesés à nouveau. On obtient ainsi par différence le poids du plumage qui est ensuite ramené au poids corporel des oiseaux saignés. A ro semaines d'âge, le poids du plumage est déterminé de même pour 2 I coquelets ( 7 de chaque génotype) du lot " chauffé " pour comparaison avec ceux du lot "témoin " de même âge.

\section{Autres mesures}

Sur les coquelets du lot " chauffé " à Io semaines d'âge, la taille des organes non emplumés est évaluée par la longueur du tarso-métatarse et la longueur des barbillons. La température rectale est mesurée à l'aide d'un thermocouple sur les coquelets (non mis à jeun) vers 9 heures du matin.

\section{4. - Analyse statistique des données}

La méthode utilisée est 1'analyse de variance avec nombres inégaux dans les sous-classes (SNEDECOR et Cochran, I967).

Quatre facteurs de variation sont contrôlés au total : sexe, génotype au locus $N a$, famille de père et environnement (caractérisé essentiellement par la température d'élevage). Cependant, du fait des contraintes matérielles et en particulier des locaux disponibles, notre dispositif expérimental ne permettait pas une analyse complète incluant l'effet et l'interaction de tous ces facteurs. Toutes les analyses par caractère ont, par suite, été faites séparément pour chaque lot et sexe, avec les facteurs contrôlés "Génotype » et "familles de père " (sauf pour le poids du plumage qui comporte une analyse avec "âge " et "génotype ", une autre avec "génotype " et " environnement").

Ce n'esi que pour les poids et gains de poids à chaque âge chez les femelles qu'une analyse de variance à 3 facteurs (génotype - famille de père - environnement) était possible. Même dans ce cas, nous avons d'abord tenu compte des analyses à 2 facteurs (génotype-famille) dans chaque lot expérimental, le but prioritaire du présent travail étant la comparaison des performances des trois génotypes dans chaque lot.

Lorsque 1'analyse de variance indique un effet significatif du facteur " génotype ", un classement des trois génotypes est fait par la méthode de SCHEFFE (KENDALI, et STUART, I967). 


\section{Résultats}

\section{I. - Croissance du plumage des coquelets}

Le tableau 3 donne les valeurs moyennes du poids du plumage (absolu ou ramené au poids corporel) par âge et génotype pour les coquelets du lot " témoin " et par génotype pour les coquelets du lot "chauffé " dont le plumage était pesé seulement à ro semaines. Le tableau 4 contient l'analyse de variance des mêmes variables par âge et génotype pour le seul lot "témoin ". Le tableau 5 présente l'analyse de variance à l'âge de ro semaines pour les coquelets des lots "témoin" et " chauffé " avec les facteurs contrôlés " génotype " et " environnement ".

\section{TA,BLEAU 3}

Poids du plumage des coquelets (brut et p. Ioo du poids corporel) par age et génotype Weight of plumage of cockevels (absolute and p. Ioo of body weight) by age and genotype

\begin{tabular}{|c|c|c|c|c|c|c|}
\hline \multirow[t]{2}{*}{ Génotype } & \multirow[t]{2}{*}{ Poids du plumage } & \multicolumn{4}{|c|}{$\begin{array}{l}\text { Lot témoin } \\
\text { Age (semaines) }\end{array}$} & \multirow{2}{*}{$\begin{array}{c}\text { Lot } \\
\text { chauffe } \\
\text { Age } \\
\text { (semaines) } \\
\text { IO }\end{array}$} \\
\hline & & 4 & 6 & 8 & IO & \\
\hline $\mathrm{NaNa}$ & $\left\{\begin{array}{l}\text { Absolu }(\mathrm{g}) \\
\text { P. Ioo du poids corporel. }\end{array}\right.$ & $\begin{array}{l}\text { I I, O } \\
4,56\end{array}$ & $\begin{array}{r}20,0 \\
4,63\end{array}$ & $\begin{array}{l}32,6 \\
3,98\end{array}$ & $\begin{array}{r}69,6 \\
4,59\end{array}$ & $\begin{array}{r}63,8 \\
5,23\end{array}$ \\
\hline$N a n a$. & $\left\{\begin{array}{l}\text { Absolu }(g) \\
\text { P. Ioo du poids corporel. }\end{array}\right.$ & $\begin{array}{l}\text { I } 2,0 \\
4,94\end{array}$ & $\begin{array}{r}24,9 \\
5,66\end{array}$ & $\begin{array}{l}4 \mathrm{I}, \mathrm{o} \\
5,08\end{array}$ & $\begin{array}{l}8 \mathrm{I}, 9 \\
5,70\end{array}$ & $\begin{array}{l}81,9 \\
6,83\end{array}$ \\
\hline na na & $\left\{\begin{array}{l}\text { Absolu }(g) \\
\text { P. I oo du poids corporel. }\end{array}\right.$ & $\begin{array}{l}\text { I } 5, \mathrm{I} \\
6,20\end{array}$ & $\begin{array}{l}30,0 \\
6,16\end{array}$ & $\begin{array}{r}54,6 \\
6,46\end{array}$ & $\begin{array}{l}99,3 \\
7,45\end{array}$ & $\begin{array}{l}88, \mathbf{I} \\
8,16\end{array}$ \\
\hline
\end{tabular}

TABLEAU 4

Coquelets lot témoin: poids du plumage par age et génotype, analyse de variance Cockevels, control group: weight of plumage by age and genotype, variance analysis

\begin{tabular}{|c|c|c|c|c|}
\hline & \multicolumn{4}{|c|}{ Source de variation } \\
\hline & Résiduelle & Génotype & Age & Interaction \\
\hline d.1. . . . . . . . . . . . . . & 70 & 2 & 3 & 6 \\
\hline Poids du plumage $(\mathrm{g})$. & 59,9 & $2361,8 * * *$ & $16301,4 * * *$ & $396,8 * * *$ \\
\hline Plumage p. Ioo du poids corporel. . & $0,3 \mathrm{I}$ & $30,63 * * *$ & $2,30^{* * *}$ & $0,92 *$ \\
\hline
\end{tabular}
variables.

Comparaison des génotypes, méthode de ScHeFFE : différences toutes significatives pour les deux

****** : Significatif respectivement au seuil $5, \mathrm{I}$ et $0, \mathrm{I}$ p. cent. 
TABLEAU 5

Poids du plumage des coquelets à ro semaines: analyse de variance facteurs " génotype " et "environnement"

Weight of plumage of cockerels at to weeks: variance analysis by genotype and environment

\begin{tabular}{|c|c|c|c|c|}
\hline & \multicolumn{4}{|c|}{ Source de variation } \\
\hline & Résiduelle & Génotype & $\begin{array}{l}\text { Environne- } \\
\text { ment }\end{array}$ & Interaction \\
\hline d.1. . . . . . . . . . . . . . . & 36 & 2 & I & 2 \\
\hline Poids du plumage $(\mathrm{g})$. & 89,2 & $4252,6 * * *$ & 320,4 & I03,5 \\
\hline Plumage p. Ioo du poids corporel . & 0,42 & $29,4^{* * *}$ & $7,2 * * *$ & 0,24 \\
\hline
\end{tabular}

$* * * * * *$ : significatif respectivem ent au seuil $5, \mathrm{r}$ et $0, \mathrm{r}$ p. roo.

\section{2. - Croissance pondérale et efficacité alimentaire: poulettes}

Aux tableaux 6 et 7 figurent les valeurs moyennes par âge et génotype pour les poids, gains de poids, consommations d'aliment et indices de consommation des femelles des lots " témoin » et "chauffé ».

\section{TABLEAU 6}

Croissance et consommation alimentaive, moyennes par génotype et ages ou périodes de deux semaines: poulettes, lot "témoin"

Growth and food consumption, means per genotype and age or 2-week period: females, control group

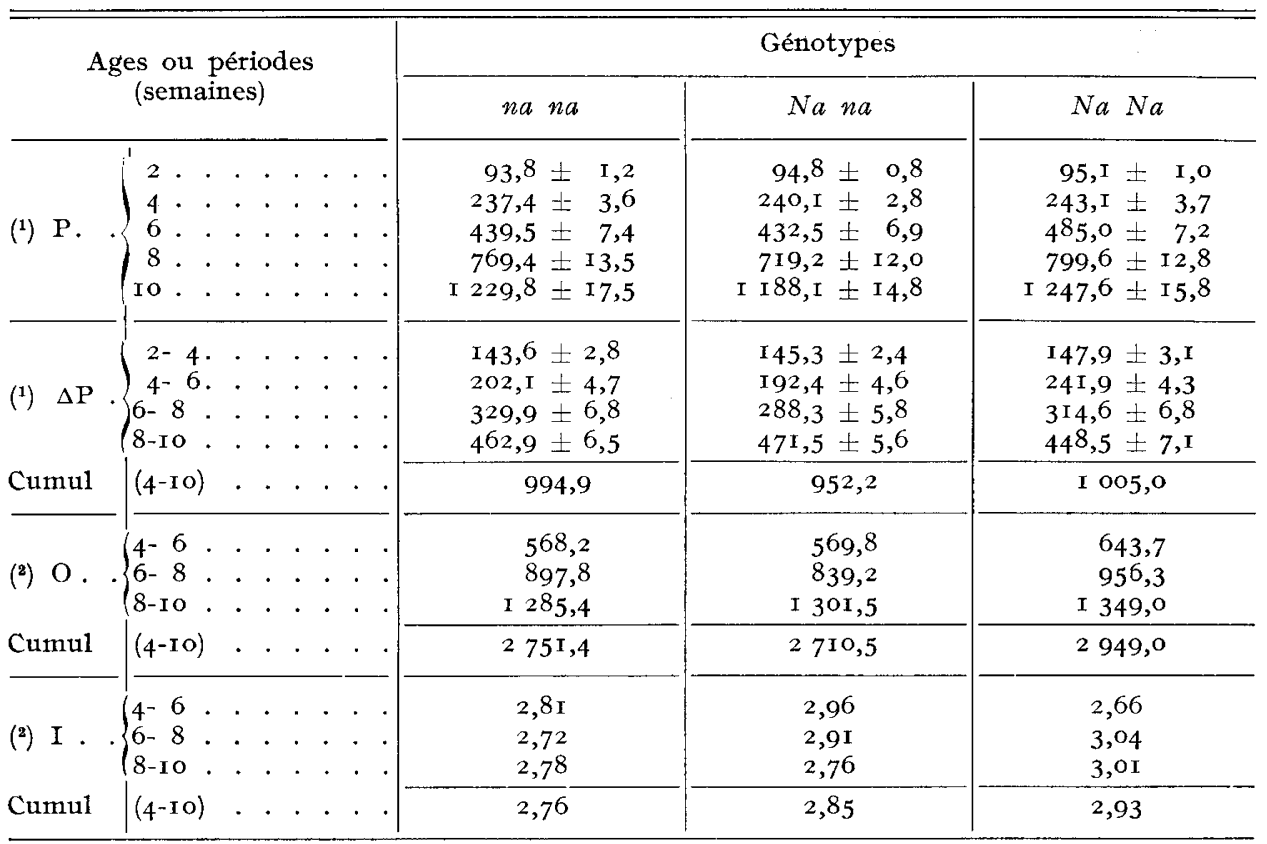

(1) A partir de données individuelles.

$\left({ }^{2}\right)$ A partir de données globales par génotype. 


\section{TABleaU 7}

Croissance et consommation alimentaire

moyennes par génotype et age ou périodes de deux semaines: poulettes, lot "chauffe" Growth and food consumption, means per genotype and age or 2-week period: pullets, heated group

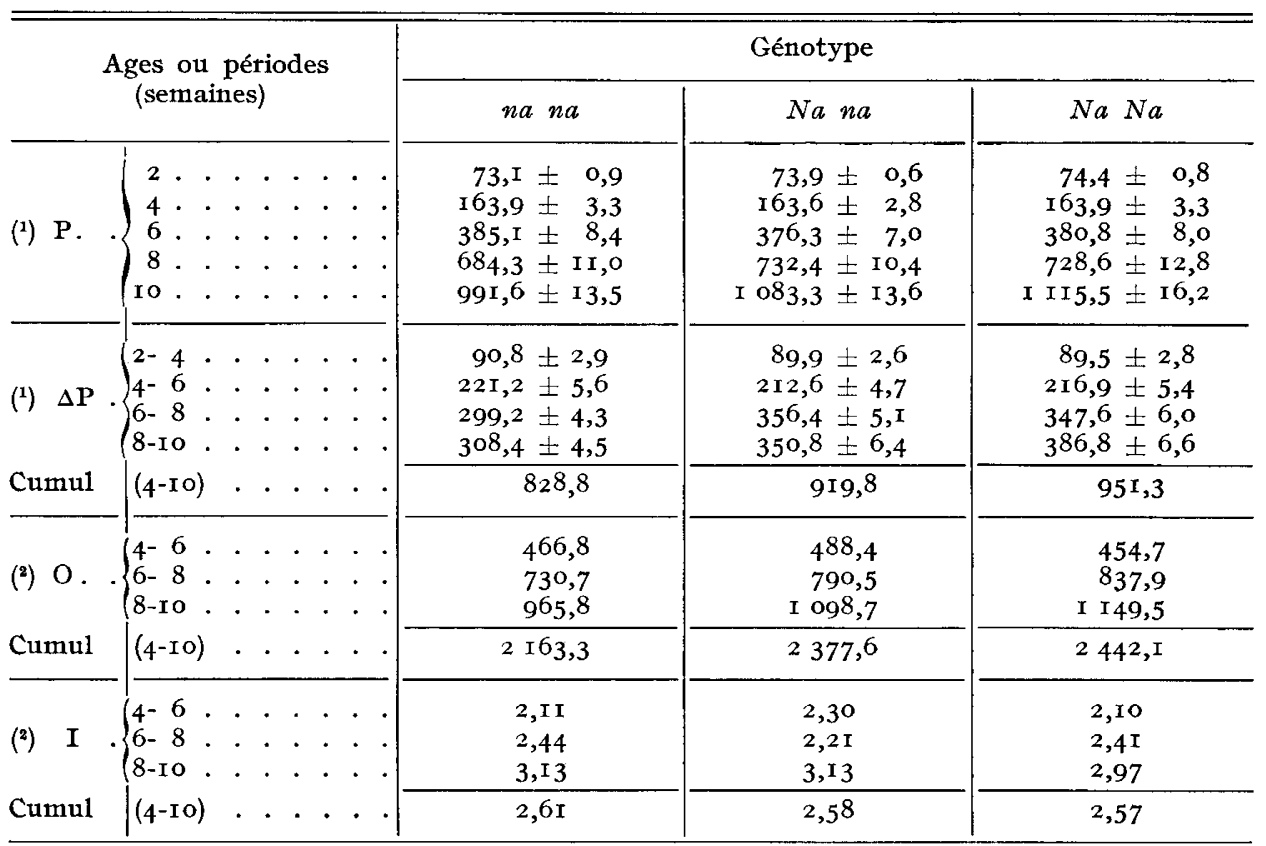

(1) A partir de données individuelles.

$\left({ }^{2}\right)$ A partir de données globales par génotype.

\section{TABLEAU 8}

Variables de croissance: poulettes, lot "témoin" analyse de variance par père et génotype

Growth variables: pullets, control group. Variance analysis according to sive and genotype

\begin{tabular}{|c|c|c|c|c|c|}
\hline \multirow{2}{*}{ Source de variation } & \multirow{2}{*}{ D.L. } & \multicolumn{4}{|c|}{ Ages ou périodes (semaines) } \\
\hline & & 4 ou $2-4$ & 6 ou $4-6$ & 8 ou $6-8$ & Io ou 8-10 \\
\hline 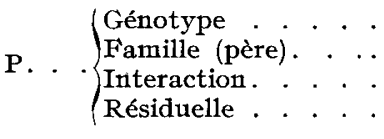 & $\begin{array}{r}2 \\
8 \\
16 \\
136\end{array}$ & $\begin{array}{l}165,1 \\
538,2 \\
377,9 \\
663,9\end{array}$ & 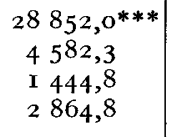 & $\begin{array}{c}64492,4^{* * *} \\
23 \text { I } 91,9^{* *} \\
6729,7 \\
8350,6(1)\end{array}$ & $\begin{array}{l}44318,6^{*} \\
287 \text { I } 9,0 \\
\text { I } 2045,0 \\
\text { I } 3 \text { I I } 4, \mathrm{I}\left({ }^{2}\right)\end{array}$ \\
\hline 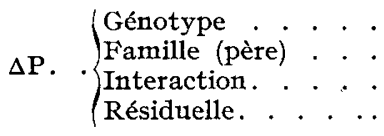 & $\begin{array}{r}2 \\
8 \\
16 \\
136\end{array}$ & $\begin{array}{r}65,8 \\
498,9 \\
318,4 \\
427,5\end{array}$ & $\begin{array}{rl}25 & 352,4^{* * *} \\
3 & 043,4^{* *} \\
& 67 \mathrm{I}, \mathrm{I} \\
\mathrm{I} & 050,4\end{array}$ & $\begin{array}{rl}28 & 851,8^{* * *} \\
8 & 179,9^{* * *} \\
2 & 837,3 \\
1 & 877,3(1)\end{array}$ & $\begin{array}{ll}6 & 546,5^{*} \\
4 & 028,3^{*} \\
2 & 440,0 \\
2 & 026, o\left({ }^{2}\right)\end{array}$ \\
\hline
\end{tabular}

******: différences significatives respectivement au seuil $5, x$ et 0,1 p. Ioo.

(1) I35 D.I...

(2) I 3 I D.I...

Comparaison des génotypes, méthođe de Scheffe : différences significatives, à l'exception de celles entre $N a n a$ et $n a$ na pour le P. $6 \mathrm{~s}$; entre $N a N a$ et $n a n a$ (P. Ios); entre $N a n a$ et $n a n a$ (P8 - Ios). 
Les tableaux 8 et 9 donnent les analyses de variance, pour chaque âge ou période, pour les deux premières variables ( $P$ et $\Delta P$ ), les deux dernières n'étant mesurées que globalement par génotype.

TABLEAU 9

Variables de croissance: poulettes, lot " ehauffe"

Analyse de variance par père et génotype

Growth variables: pullets, heated group. Variance analysis according to sire and genotype

\begin{tabular}{|c|c|c|c|c|c|c|c|c|}
\hline \multirow{2}{*}{\multicolumn{4}{|c|}{ Source de variation }} & \multirow{2}{*}{ D.L. } & \multicolumn{4}{|c|}{ Ages ou périodes } \\
\hline & & & & & 4 ou $2-4$ & 6 ou $4^{-6}$ & 8 ou $6-8$ & ro ou 8-ro \\
\hline $\mathbf{P}$ & - $\left\{\begin{array}{l}\text { Génotype } \\
\text { Famille (père) } \\
\text { Interaction. } \\
\text { Résiduelle }\end{array}\right.$ & 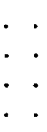 & $\begin{array}{c}\cdot \\
\cdot \\
\cdot \\
\cdot \\
\cdot\end{array}$ & $\begin{array}{r}2 \\
8 \\
16 \\
\mathrm{I} 37\end{array}$ & $\begin{array}{c}\mathrm{I} 6,2 \\
269 \mathrm{I}, 8 * * * \\
244,8 \\
450,6\end{array}$ & $\begin{array}{rl} & 870,0 \\
\text { I } 3 & 670,4 * * * \\
\text { I } 709,4 \\
2906,4\end{array}$ & $\begin{array}{c}34088,3^{* *} \\
19167,5^{* *} \\
6101,0 \\
6723,9\end{array}$ & $\begin{array}{l}\text { I9I } 906,4^{* * *} \\
\text { 20 } 5 \text { I } 4,8 \\
\text { II } 880, \text { I } \\
\text { I I } 023,7\left(^{1}\right)\end{array}$ \\
\hline$\Delta \mathrm{P}$. & $\left\{\begin{array}{l}\text { Génotype } \\
\text { Famille } \cdot \\
\text { Interaction. } \\
\text { Résiduelle } .\end{array}\right.$ & $\begin{array}{ll}\cdot & \cdot \\
\cdot & \cdot \\
\cdot & \cdot\end{array}$ & $\begin{array}{ll}\cdot & \cdot \\
\cdot & . \\
. & . \\
\cdot & .\end{array}$ & $\begin{array}{r}2 \\
8 \\
16 \\
\mathrm{r} 37\end{array}$ & $\begin{array}{c}64,9 \\
235,8 * * * \\
190,7 \\
328,0\end{array}$ & \begin{tabular}{ll|} 
& 942,4 \\
4 & $94 \mathrm{I}, 3 * * *$ \\
I & $3 \mathrm{I} 3,0$ \\
I & $333, \mathrm{I}$
\end{tabular} & $\begin{array}{rl}45 & 396,0^{* * * *} \\
\text { I } & 224,8 \\
\text { I } & 803,5 \\
\text { I } & 468,0\end{array}$ & $\begin{array}{rl}68 & 096,5^{* * *} \\
2 & 800,0 \\
\text { I } 842,5 \\
\text { I } 842,2\left({ }^{1}\right)\end{array}$ \\
\hline
\end{tabular}

******: Différence significative respectivement au seuil $5, \mathrm{r}$ et $0, \mathrm{r}$ p. roo.

(1) D.I. $=136$.

Comparai son des génotypes, méthođe de Schefré : différences toutes significatives, à l'exception de celle entre $\mathrm{Na} N a$ et $\mathrm{Na}$ na pour le poids à $8 \mathrm{~s}$.

\section{TABLEAU IO}

Poids et gains de poids des poulettes: Analyse de variance à 3 facteurs (génotype-famille-environnement) pour chaque age ou période

Body weight and weight gain of pullets: 3-way analysis of variance (genotype-sive familyenvironment) for each age or period

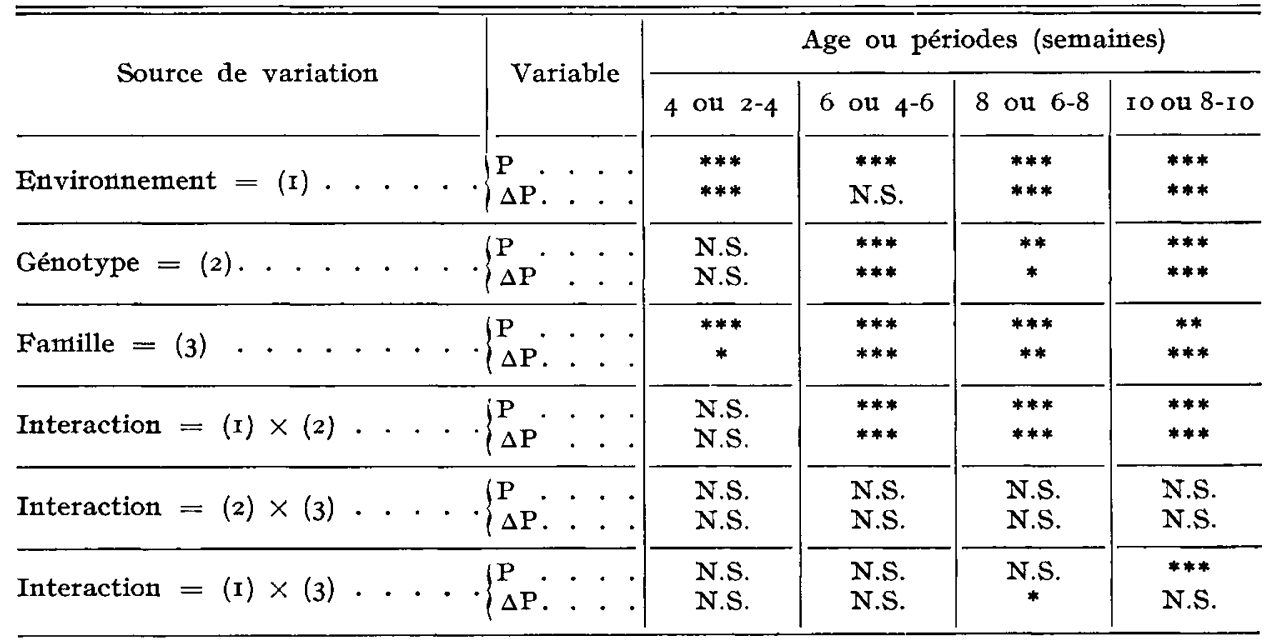

*****: Différence significative respectivem ent au seuil $5, \mathrm{r}$ et $0, \mathrm{x}$. roo. 
Le tableau ro concerne l'analyse de variance à 3 facteurs (génotype-familleenvironnement) sur les mêmes variables. Il présente le degré de signification des variances.

\section{3. - Croissance et efficacité alimentaire: coquelets}

Le tableau II indique les valeurs moyennes par âge et génotype pour les quatre variables $P, \Delta P, O$ et $I$, pour les coquelets " chauffés " en cages individuelles. Le tableau $\mathrm{I} 2$ contient les analyses de variance correspondantes.

\section{TABLEAU II}

Moyennes par génotypes et par âges ou période de 2 semaines:

coquelets, lot "chauffe"

Means per genotype and age or 2-week period: cockerels, heated group

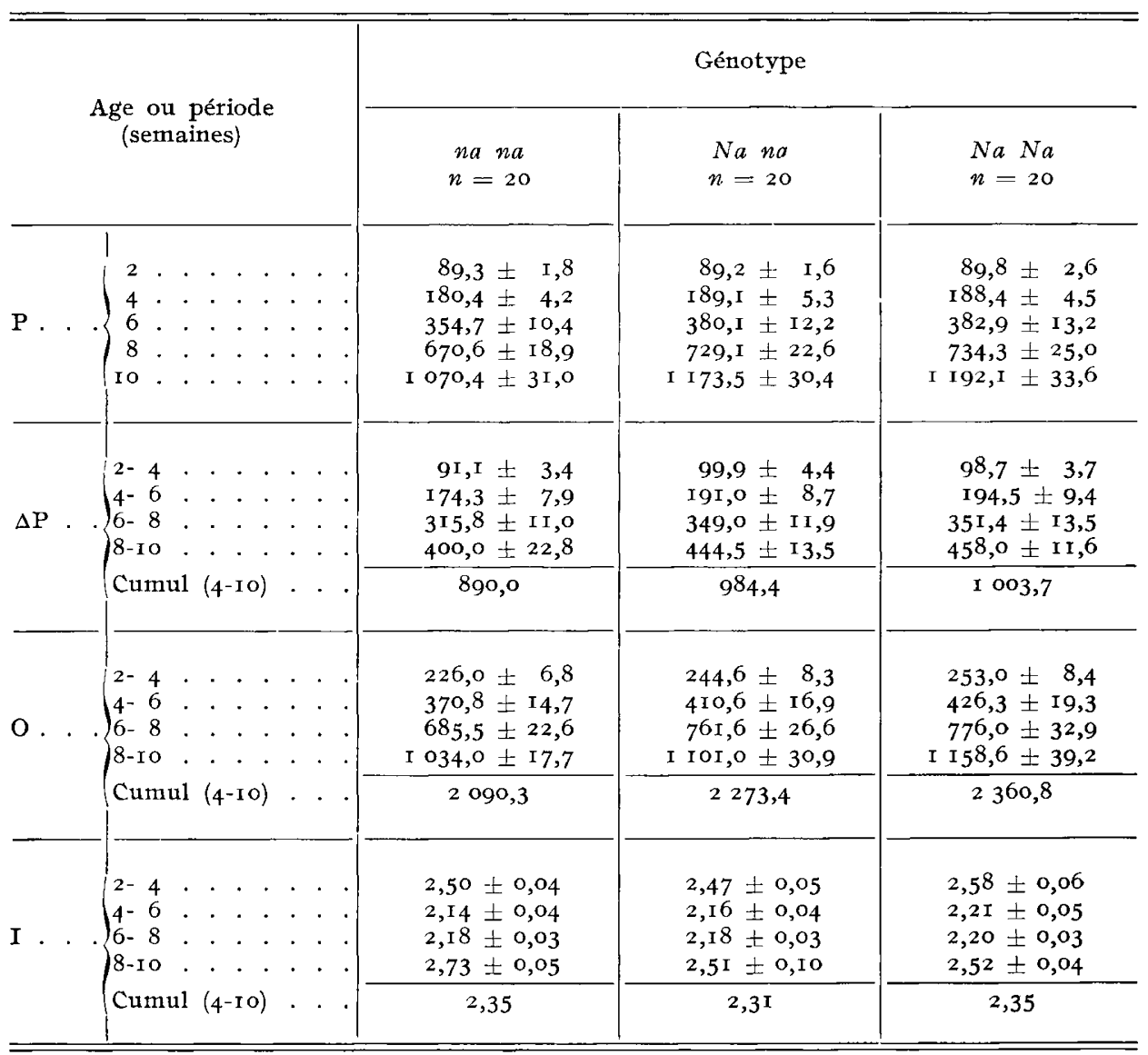




\section{TABLEAU I2}

Coquelets lot "chauffe": analyse de variance par génotype et famille aux divers âges Cockerels, heated group : variance analysis according to genotype and sive family, for each age

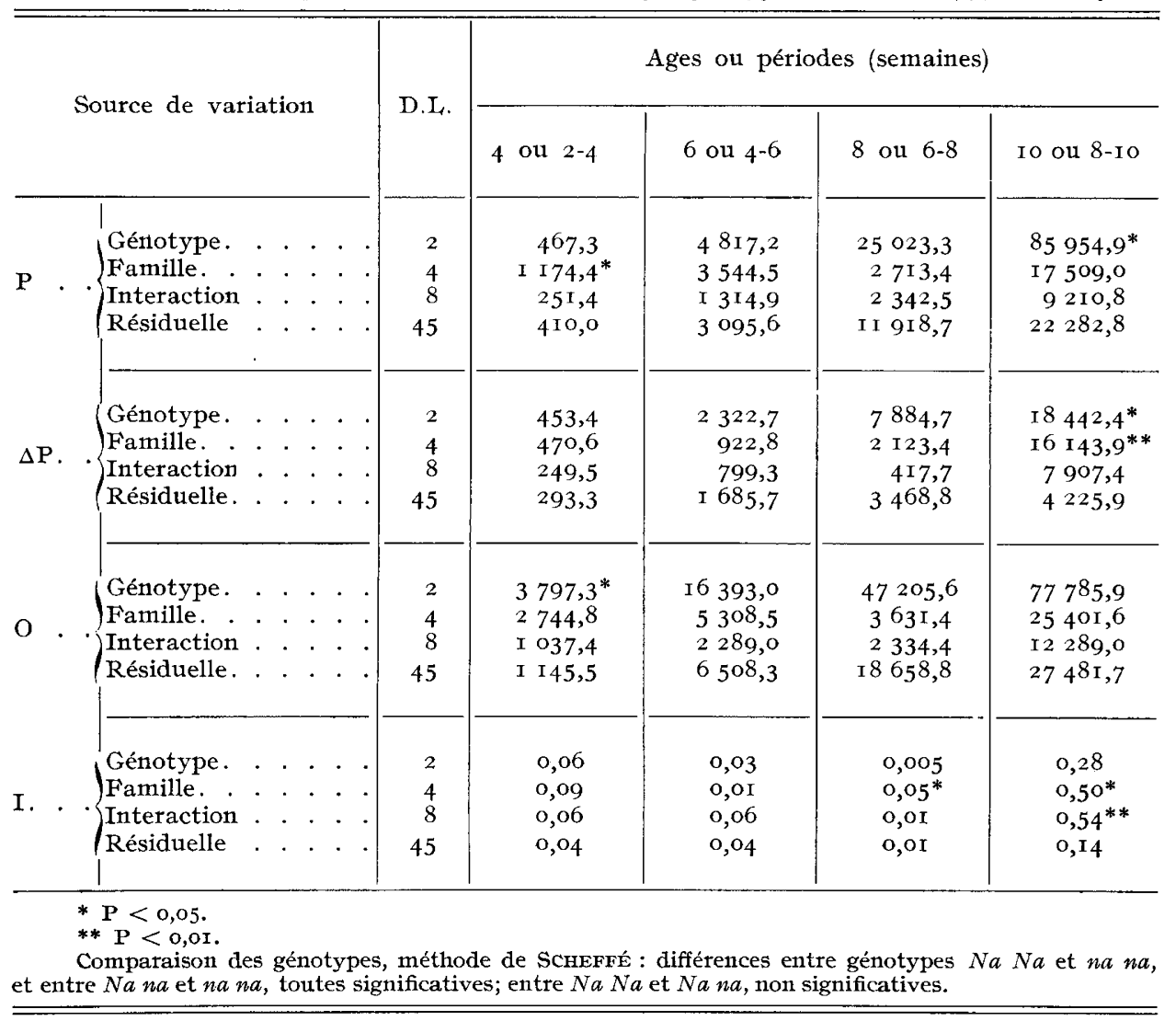

\section{TABLEAU I3}

Longueur des tarses et des barbillons, température rectale: moyennes par génotype coquelets lot "chauffe " à $T$ o semaines ( $\mathrm{n}=20$ par génotype)

Shank length, wattle length, rectal temperature: means per genotype cockerels of heated group at Io weeks $(\mathrm{n}=20$ per genotype)

\begin{tabular}{|c|c|c|c|}
\hline \multirow{2}{*}{ Génotype } & \multicolumn{3}{|c|}{ Caractères } \\
\hline & $\begin{array}{l}\text { Long. Barbillons } \\
\qquad(\mathrm{mm})\end{array}$ & $\begin{array}{l}\text { Long. Tarses } \\
\text { (mm) }\end{array}$ & Temp. Rectale \\
\hline 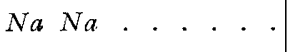 & $34,0 \pm 1,6$ & $105,6 \pm \mathrm{I}, \mathrm{O}$ & $4 \mathrm{I}, 4 \pm 0,07$ \\
\hline$\cdot \cdot \cdot \cdot \cdot \cdot \cdot$ & $30,5 \pm \mathrm{I}, 7$ & $\mathrm{IO} 4,2 \pm \mathrm{I}, \mathrm{O}$ & $41,6 \pm 0,06$ \\
\hline na na......... & $28,6 \pm \mathrm{I}, 4$ & $\mathrm{IO} 2,6 \pm 0,8$ & $4^{1,7} \pm 0,05$ \\
\hline
\end{tabular}




\section{4. - Autres variables (sur coquelets, lot "chauffé ")}

Les tableaux I3 et I4 contiennent respectivement les valeurs moyennes par génotype et les analyses de variance pour les deux mensurations corporelles et la température rectale à Io semaines.

\section{TABLEAU I4}

Longueur des tarses et des Barbillons, température rectale: coquelets lot "chauffe" Analyse de variance par génotype et famille

Shank length, wattle length, rectal temperature: cockerels of heated group Variance analysis according to genotype and sive family

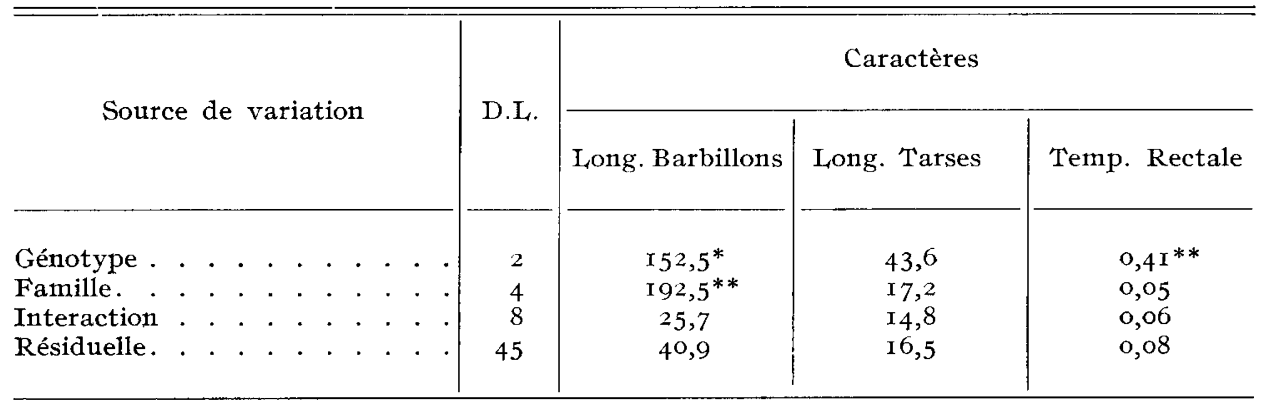

$* \mathrm{P}<0,05$.

** $\mathrm{P}<0$,or.

Comparaison des génotypes, méthode de SchefFÉ : différences toutes significatives entre $N a \mathrm{Na}$ et $N a n a$ ou $n a n a$; non significatives entre $N a$ na et $n a$ na.

\section{Discussion}

\section{I. - Croissance du plumage}

L'analyse de variance et les tests de ScheFfe (tab1. 4) montrent que les trois génotypes sont significativement distincts pour le poids du plumage, tant en valeur absolue qu'en pourcentage du poids corporel. Ce résultat, conforme à celui de Bordas et al. (r978), s'accorde aussi avec les conclusions de CRAwFord (r976) et SCOTT et CRAWFORD (r977) sur la dominance incomplète du gène " cou nu ».

L'influence significative de l'âge sur le poids du plumage en valeur absolue se comprend aisément. De plus, on note un effet significatif de l'âge sur la quantité du plumage ramenée au poids du corps, avec une tendance à l'accroissement. L'interaction génotype $\times$ âge sur le poids absolu du plumage n'a pas une interprétation évidente. L'interaction des mêmes facteurs sur le poids du plumage en p. Ioo du poids corporel représente une évolution différentielle de ce paramètre suivant le génotype. L'accroissement relatif du plumage est de moins en moins marqué quand on passe des coquelets normalement emplumés aux hétérozygotes, puis aux homozygotes "cou nu. 
Quant à la température d'élevage, le tableau 5 montre qu'elle n'influe pas significativement sur le poids absolu du plumage. Par contre, son effet sur le rapport du poids du plumage au poids de l'oiseau est hautement significatif dans l'ensemble, en accord avec l'effet dépressif de la température élevée sur le poids corpore1, de sorte qu'à poids égal les poulets sont mieux emplumés en lot chauffé.

\section{2. - Croissance corporelle, efficacité alimentaire et autres mesures}

\section{- Poulettes au sol à température normale (lot " témoin ": tab1. 6 et 8 )}

Entre 4 et 6 semaines, les poulettes $N a N a$ se développent significativement plus vite que les deux autres génotypes, non différents significativement. Cette période ayant correspondu à des températures ambiantes relativement élevées (tabl. I), le génotype $N a N a$ a pu bénéficier de sa capacité plus grande de thermolyse. Il a consommé plus d'aliment avec une relativement bonne efficacité; par contre les poulettes $\mathrm{Na} n a$ ont une efficacité alimentaire apparemment inférieure à celle des poulettes na na.

De 6 à 8 semaines, la température ambiante est plus basse. Ceci coïncide avec un taux de croissance légèrement plus élevé chez les poulettes na na, et peu différent pour les deux autres génotypes. Les poulettes na na ont la meilleure efficacité alimentaire et les homozygotes $N a N a$ la moins bonne, avec la plus grande quantité d'aliment consommée. Cependant, à 8 semaines, le poids moyen du génotype $\mathrm{Na} N a$ reste encore le plus élevé.

A ro semaines, le poids moyen des poulettes na na ne diffère plus significativement de celui des poulettes $N a N a$; les hétérozygotes $N a n a$ ont une taille légèrement inférieure. $D$ 'autre part, globalement et malgré 1'absence de tests de signification, il paraît clair que la consommation d'aliment est la plus élevée et l'indice de consommation le moins bon pour le génotype $N a N a$. La consommation diffère peu entre les deux autres génotypes, mais l'indice est meilleur pour les individus na na.

L'ensemble de ces résultats paraît interprétable par 1'hypothèse selon laquelle les déperditions caloriques supérieures des poulets moins emplumés nécessitent en compensation une plus grande ingestion de nutriments énergétiques, sans qu'au total la croissance en soit modifiée de façon importante.

\section{- Poulettes au sol à température élevée (lot "chauffé »: tabl. 7 et 9)}

Dans le lot "chauffé ", on observe jusqu'à 6 semaines une croissance équivalente dans les 3 génotypes, une consommation alimentaire peu différente, et une efficacité légèrement inférieure chez les poulettes $N a$ na. De 6 à ıo semaines, $1 \mathrm{a}$ consommation alimentaire moyenne est la plus basse dans le génotype na na; le génotype $\mathrm{Na} \mathrm{Na}$ est sensiblement supérieur à $\mathrm{Na}$ na. De 8 à ro semaines, les poulettes normalement emplumées consomment environ I6 et 12 p. Ioo de moins, respectivement, que les poulettes $N a N a$ et $N a$ na. Corrélativement, le taux de croissance moyen du génotype na na est significativement inférieur à celui des deux autres génotypes, de 6 à 8 et de 8 à ro semaines, et le génotype $N a$ na est légèrement supérieur au génotype $\mathrm{Na} N a$. 
A l'âge de Io semaines, le poids moyen diffère significativement entre les 3 génotypes. Les poulettes normalement emplumées ont un retard de croissance de $92 \mathrm{~g}$. et $\mathrm{I} 24 \mathrm{~g}$ par rapport aux individus $N a$ na et $N a N a$, soit 8,5 et Ix,o p. roo respectivement. L'efficacité alimentaire globale de 4 à Io semaines est voisine pour les trois génotypes.

\section{- Comparaison des poulettes dans les deux environnements}

Le tableau ro montre une interaction hautement significative entre génotype et environnement pour les poids corporels et gains de poids après 6 semaines d'âge. En fait, le classement relatif des gains de poids moyens des deux homozygotes après 6 semaines s'inverse selon la température, et le génotype hétérozygote est en moyenne intermédiaire. Les environnements diffèrent, outre la température, par deux facteurs secondaires, jour d'éclosion et intensité de l'éclairement; mais il fait peu de doute que, concernant la croissance, la température est de loin le facteur prépondérant. L'effet dépressif bien connu des températures élevées sur les gains de poids est illustré ici, dès l'âge de 2 semaines. La comparaison des tableaux 6 et 7 montre en outre que l'indice de conversion alimentaire, dans l'ensemble est meilleur en lot chauffé jusqu'à 1'âge de 8 semaines, puis en lot témoin de 8 à to semaines. Cet effet de l'âge suggéré par nos données serait à confirmer. Des travaux antérieurs (p. ex. Howes et al., r962; Orson et al., r970), indiquant un meilleur indice de transformation à température élevée, se rapportent à des âges précoces, comme dans la prrésente expérience.

\section{- Coquelets en cages individuelles à température élevée (lot " chauffé ")}

On observe (tab1. II) dès après 1'âge de 4 semaines, que la consommation alimentaire des coquelets normalement emplumés $(n a n a)$ est en moyenne significativement inférieure à celle des deux autres génotypes. Jusqu'à 1'âge de ro semaines, la quantité d'aliment ingérée reste plus élevée dans les deux derniers groupes. L'analyse de variance après 4 semaines n'indique pas de différences significatives entre génotypes; cependant les tests $t$ montrent une différence significative entre homozygotes. Cumulée de 2 à ro semaines, la consommation alimentaire moyenne individuelle des coquelets $N a N a$ excède de II,5 et 3,7 p. Ioo respectivement celle des génotypes $n a$ na et $N a$ na. Dans la même période, les coquelets $N a N a$ et $N a n x$ ont sur les individus normalement emplumés un avantage de croissance qui augmente avec l'âge. A Io semaines, les homozygotes " cou nu " pèsent Io,2 p. Ioo de plus que les coquelets na na et seulement I,6 p. Iоo de plus que les hétérozygotes.

Pour la longueur des tarses à Io semaines, les différences entre génotypes sont faibles; elles correspondent apparemment aux différences de taille moyenne à cet âge. Quant à la longueur des barbillons, elle est notablement plus grande pour les individus " cou nu " (l'hétérozygote étant intermédiaire), ce qui peut être un léger avantage supplémentaire vis-à-vis de la chaleur en augmentant la déperdition calorique par ces organes.

Les différences de température rectale, quoique faibles, sont significatives. Ce résultat paraît aller dans le sens de ceux de BERMAN (I973) montrant une corrélation négative entre variation de température rectale et croissance en ambiance chaude. Il confirme la meilleure tolérance vis-à-vis de la claleur des homozygotes " cou nu ". 


\section{Conclusion}

Tous les résultats présents, comme ceux précédemment obtenus (BoRDAs et al., I978), paraissent converger pour indiquer que la suppression partielle de l'emplument par le gène "cou nu " améliore la thermotolérance en période de croissance, l'animal se trouvant moins contraint de réduire sa consommation alimentaire et ayant, corrélativement, une croissance plus rapide avec un indice de transformation équivalent à âge égal.

Nos résultats suggèrent que, sous des conditions de température et d'hygrométrie identiques à celles réalisées ici (un peu au-dessus de $30^{\circ} \mathrm{C}$ durant toute la croissance, avec hygrométrie faible à modérée), la fixation du gène " cou nu " à l'état homozygote dans des populations destinées à la production du poulet de chair apporterait un gain de croissance supérieur à Io ou II p. Ioo suivant le sexe, un indice de consommation légèrement amélioré à âge égal (mais nettement supérieur à poids fixé), ainsi qu'une augmentation de l'ordre de $3 \mathrm{p}$. Ioo du rendement à l'abattage par suite de la réduction du plumage. Il est intéressant, qu'à température élevée, l'hétérozygote $N a n a$, pour la croissance, se situe, dans les deux sexes, plus près de l'homozygote " cou nu " (entre +8 et +9 p. Ioo par rapport à na na à Io semaines), avec un rendement à l'abattage amélioré d'environ I,5 p. I0o. La seule fixation du gène $N a$ dans une lignée mâle permettrait déjà, dans ces conditions, un gain notable en croisement de première génération. I1 faudrait voir dans quel éventail de températures ambiantes (variables ou non) et de degrés hygrométriques ces avantages attachés au gène $N a$ resteraient significatifs. La même question peut être posée pour l'aliment, dont le taux protéique, dans la présente expérience, ne permettait vraisemblablement pas d'atteindre la croissance maximum possible en lot chauffé.

Nos résultats paraissent analogues à ceux obtenus par JoHnson et Somes (I978) avec le gène sc (scaleless).

Reçu pour publication en février 1980.

\section{Summary}

\section{Naked neck gene and growth performance of chicks according to ambient temperature}

Growth performance was compared for pullets of the genotypes $\mathrm{Na} \mathrm{Na}$ (" naked neck" homozygotes), $\mathrm{Na} n a$ (" naked neck" heterozygotes) and na na (normal plumage). They were reared in floor pens, at ambient temperature close to $20^{\circ} \mathrm{C}$ from 4 weeks of age (" control ") or $3 \mathrm{I}^{0} \mathrm{C}$ till $\mathrm{ro}$ weeks of age (" heated " group). A similar comparison at $3 \mathrm{I}^{\circ} \mathrm{C}$ till io weeks was done on cockerels of the same genotypes in individual cages. Chicks were weighed every 2 weeks. Food consumed was measured for the same periods (total per genotype for females, individually for males). On males at ro weeks, wattle length, shank length, rectal temperature and weight of plumage were recorded. Plumage was weighed at successive ages in another group of males.

Absolute as well as relative plumage weight differs significantly for the three genotypes. Its relative increase with age seems less for "naked neck" chicks. Body weight at ro weeks is not significantly different for pullets of the three genotypes in the control group, but food intake is higher and food efficiency is inferior for the $\mathrm{Na} \mathrm{Na}$ genotype. In the " heated "group, $\mathrm{Na} \mathrm{Na}$ birds at Io weeks have a growth advantage over $n a n a$ chicks of about I I $\mathrm{p}$. roo for females and ro p. Ioo for males; similarly, heterozygotes have in both sexes an advantage of about 
9 p. roo. Food consumed is higher for , naked neck " birds; food conversion shows little difference between genotypes in these conditions. Rectal temperature at Io weeks in the heated group is slightly lower for $N a N a$ or $N a$ na than for na na males. These results show that reduction of feathering by the $N a$ gene improves heat tolerance in the growing period. This, in addition to the higher slaughter yield of $N a N a$ and $N a n a$ chicks compared to those of na na genotype (resp. by 3 and $\mathrm{r} .5$ p. Ioo )because of the plumage reduction, suggests that, at temperature regimens like that realized in our , heated " group, the introduction of the " naked neck" gene in broiler-type populations may be advantageous.

\section{Références bibiographiques}

Berman A., 1973. Homeothermy and growth rate in the fowl. Brit. Poult. Sci., 14, 319-328. Bordas A., MERat P., Sergent D., RiCard F. H., I978. Influence du gène $N a$ (" cou nu ") sur la croissance, la consommation alimentaire et la composition corporelle du poulet selon la température ambiante. Ann. Génét. Sél. anim., 10, 209-23I.

CRAWFORD R. D., I976. Incomplete dominance of the gene for Naked Neck in the domestic fowl. Poult. Sci., 55, 820-822.

Howes J. R., GRUB W., Rollo C. A., r962. The effects of constant high temperature regimes upon broiler growth, feed efficiency, body composition and carcass quality. Poult. Sci., 41, I 652 (abstr.).

HuT' F. B., I949. Genetics of the fowl. McGraw Hill, New York.

Johnson S., Somes R. G. Jr., 1978. The effect of the scaleless gene on the performance of broilers in a hot climate. $C$. $R$. I $6^{\mathrm{e}}$ congrès mondial d'aviculture, 779-786.

KENDALI, M. G., STUART A., I 967 . The advanced theory of statistics. Griffin, London.

MERAT P., I979. Effets associés au gène $\mathrm{Na}$ (" cou nu ") sur le poids corporel et le poids des œufs chez des poules "normales" et "naines". Ann. Génét. sél. anim., 11, I27-I3r.

OLSON D. W., BIRD H. R., SUNDE M. I., I970. Effect of temperature and humidity on the growth of chickens and on the metabolizable energy of feedstuffs. Poult. Sci., 49, 1422 (abstr.).

SCOTT T., CRAWFORD R. D., I977. Feather number and distribution in the throat tuft of naked neck chicks. Poult. Sci,, 56, 686-688.

SMITH T., LEE R., I977. A study of the Naked neck gene of fow1. Poult. Sci., 56, I $75^{8}$ (abstr.).

SNedefor G. W., Cochran W. G., I967. Statistical methods. Iowa State University Press, Ames. 